\title{
Analysis of linear two-dimensional general rate model for chromatographic columns of cylindrical geometry
}

\author{
Shamsul Qamar ${ }^{\mathrm{a}, \mathrm{c}, *}$, David U Uche ${ }^{\mathrm{a}}$, Farman U Khan ${ }^{\mathrm{a}, \mathrm{b}}$, Andreas Seidel-Morgenstern ${ }^{\mathrm{c}}$ \\ ${ }^{a}$ Department of Mathematics, COMSATS Institute of Information Technology, \\ Park Road Chak Shahzad Islamabad, Pakistan \\ ${ }^{b}$ Department of Mathematics, HITEC University Taxila Cantt, Pakistan \\ ${ }^{c}$ Max Planck Institute for Dynamics of Complex Technical Systems, Magdeburg, Germany
}

\section{Abstract}

This work is concerned with the analytical solutions and moment analysis of a linear twodimensional general rate model (2D-GRM) describing the transport of a solute through a chromatographic column of cylindrical geometry. Analytical solutions are derived through successive implementation of finite Hankel and Laplace transformations for two different sets of boundary conditions. The process is further analyzed by deriving analytical temporal moments from the Laplace domain solutions. Radial gradients are typically neglected in liquid chromatography studies which are particularly important in the case of nonperfect injections. Several test problems of single-solute transport are considered. The derived analytical results are validated against the numerical solutions of a high resolution finite volume scheme. The derived analytical results can play an important role in further development of liquid chromatography.

Key words: Liquid chromatography, cylindrical column, general rate model, analytical solutions, moment analysis, mass transfer.

\section{1. introduction}

Mathematical modeling of liquid chromatography has been an attractive field of research since the 1960s, leading to a more efficient use of chromatographic columns. The approach provides important information about physical and thermodynamical kinetics as well as

\footnotetext{
${ }^{*}$ Corresponding author. Tel: +49-391-6110454; fax: +49-391-6110500 Email addresses: qamar@mpi-magdeburg.mpg.de (Shamsul Qamar)
} 
flow phenomena through packed-beds. Understanding of the effects of operating variables and parameters characterizing the column is needed for an accurate theoretical analysis of the elution profiles and to optimize the operating conditions [1-3]. Different mathematical models exist in the literature describing the chromatographic process. The most notable amongst them are the general rate model (GRM), the equilibrium dispersive model (EDM), and the lumped kinetic model (LKM) [1-9]. All these models need important input information regarding the thermodynamic equilibrium of the distribution of the components between the mobile and stationary phases. They differ essentially regarding the consideration of unavoidable mass transfer processes, which cause undesired band broadening $[1,2]$.

In the literature, analytical solutions for one-, two- and three-dimensional advectiondispersion equations (ADEs) have been developed for predicting the transport of various contaminants in the soil. The analytical solutions of the one-dimensional ADE subject to various initial and boundary conditions were derived in [10]. The analytical solutions of the two-dimensional ADE with various source boundary conditions were presented in $[11,12]$. The analytical solutions for three-dimensional ADE were derived in $[13,14]$. However, these models were mostly limited to ADE in Cartesian coordinates describing steady uniform flow [14]. The analytical solutions of the two-dimensional ADE in cylindrical coordinates are particularly useful for analyzing problems of the two-dimensional solute transport in a porous medium system with steady uniform flow [14-19].

In the liquid chromatography, the analytical solutions and moment analysis of the onedimensional EDM, LKM and GRM have been derived for linear isotherms using the Laplace transformation [4-9, 20, 21]. Very recently, we have derived analytical solutions and temporal moments of linear 2D-EDM and 2D-LKM for simulating liquid chromatography in cylindrical columns [22-24]. This article extends those analysis to linear 2D-GRM. Moment analysis is useful and effective technique for deducing important information about the retention equilibrium and mass transfer kinetics in a fixed-bed column. The moment generating property of the Laplace domain solutions can be used to derive analytical temporal moments. These moments can be used to get important information about the 
retention times, band broadenings, and front asymmetries. Several authors have derived moments for various boundary conditions (BCs) [2, 5-8, 20, 21, 25-37].

In this article, the above analysis is further extended by analytically solving a 2D-GRM through simultaneous implementation of Hankel and Laplace transformations. In the current scenario, no analytical Laplace inversion is possible. Therefore, numerical Laplace inversion is applied to get back semi-analytical solutions in the actual time domain [38]. To analyze the effects of different kinetic parameters, statistical temporal moments are derived from the Hankel and Laplace transformed solutions. A high resolution upwind finite-volume scheme (HR-FVS) is extended to numerically approximate the current model equations $[39,40]$. To illustrate the potential of current analysis, several case studies are carried out considering a wide range of mass transfer kinetics. Moreover, relations are derived for matching the first two moments of 2D-GRM and simplified 2D-LKM. The derived semi-analytical results are critically checked against the numerical solutions of suggested HR-FVS.

The novelty of this article specifically include: (a) the derivation of analytical solutions of linear 2D-GRM for two different sets of boundary conditions, (b) injection of specific profiles to amplify the effect of possible rate limitations of the mass transfer in the radial direction, (c) derivation of useful moment expressions, (d) implement of a numerical scheme to the model equations, and (e) derivation of relations among the kinetic parameters of 2D-GRM and 2D-LKM through comparison of their respective moments. The derived analytical and numerical solutions are useful tools for further developments in the liquid chromatography. For instance, this analysis can be used for studying the effects of mass transfer kinetics on the elution profiles, for sensitivity analysis, for validating numerical solutions, and for determining longitudinal and radial dispersion coefficients from experimentally determined elution profiles, among others. The studied 2D-model is more general and flexible than the classical 1D-models [7].

The current 2D-model can be useful if (i) the injection at the column inlet is not perfect (i.e. a radial profile is introduced at the column inlet), (ii) the column is not homogeneously packed (which is more probable for larger columns), (iii) there are radial temperature 
gradients, which are connected also with radial concentration gradients. All such scenario can happen in reality. In many chromatography processes deviations from predictions using a simpler 1D model might be small. However the differences are difficult to evaluate and it is desirable to have quantitative tools and criteria to rationally select the right model. With our current 2D-GRM model, we can study the situation (i) by assuming injections in inner cylindrical core or outer annular region. Situations (ii) and (iii) are more complicated and require further model extensions, for example we have to consider variable column porosities and to include energy balance equation in the current model equations. Such extensions require more detailed treatment which is outside the scope of this paper.

The remaining parts of this article are organized as follows. In Section 2, the linear 2DGRM model is introduced. In Section 3, the analytical solution of 2D-GRM for considered two types of boundary conditions are derived. In Section 4, analytical temporal moments are derived. In Section 5, the numerical test problems are presented. Lastly, concluding remarks are given in Section 6 .

\section{The mathematical model of 2D-GRM}

In liquid chromatography, the 2D-GRM considers several contributions of the mass transfer processes that lead to band broadening. Let $t$ denotes the time coordinate, $z$ represents the axial coordinate along the column length and $\rho$ is the radial coordinate along the column radius. The solute travel along the column axis in the $z$-direction by advection and axial dispersion and spreads along the column radius in the $\rho$-direction by radial dispersion. The following particular injection conditions are assumed to amplify the effects of mass transfer in the radial direction. The inlet cross section of the column is divided into an inner cylindrical core and an outer annular ring (see Figure 1) by introducing a new parameter $\bar{\rho}$. The injection can be done either through an inner core, an outer ring or through the whole cross section. The latter case results if $\bar{\rho}$ is set equal to the radius of the column denoted by $R$. Since in the latter case no initial radial gradients are provided, the solutions should converge into the solution of the simpler one-dimensional model [7]. It is, however, 
important to mention that probably the practical relevance of such kind of injections is of minor importance.

The mass balance equation for a single-solute percolating through a cylindrical column of radius $R$ filled with spherical particles of radius $R_{p}$ is given as

$$
\frac{\partial c}{\partial t}+u \frac{\partial c}{\partial z}=D_{z} \frac{\partial^{2} c}{\partial z^{2}}+D_{\rho}\left(\frac{\partial^{2} c}{\partial \rho^{2}}+\frac{1}{\rho} \frac{\partial c}{\partial \rho}\right)-\frac{3}{R_{p}} F k_{\text {ext }}\left(c-c_{p}\left(r_{p}=R_{p}\right)\right) .
$$

In the above equation, $c$ is the concentration of a solute in the bulk phase of the fluid, $c_{\mathrm{p}}$ is the concentration of the solute in the pores of the particles, $u$ is the interstitial velocity, $D_{\mathrm{z}}$ is the axial dispersion coefficient, and $F$ is the phase ratio which is defined in term of the external porosity $\epsilon_{b}$ as $F=\left(1-\epsilon_{b}\right) / \epsilon_{b}$. Moreover, $D_{\rho}$ represents the radial dispersion coefficient, $k_{\text {ext }}$ is the external mass transfer coefficient and $r_{p}$ is the radial coordinate of spherical particles.

The mass balance equation in the pores of the particles, considering two mechanisms of intraparticle transport, can be expressed as

$$
\epsilon_{p} \frac{\partial c_{p}}{\partial t}+\left(1-\epsilon_{p}\right) \frac{\partial q_{p}^{*}}{\partial t}=\frac{1}{r_{p}^{2}} \frac{\partial}{\partial r}\left[r_{p}^{2}\left(\epsilon_{p} D_{p} \frac{\partial c_{p}}{\partial r_{p}}+\left(1-\epsilon_{p}\right) D_{s} \frac{\partial q_{p}^{*}}{\partial r_{p}}\right)\right]
$$

where, $q_{p}^{*}$ is local equilibrium concentration of the solute in the stationary phase, $D_{p}$ is the pore diffusivity, $\epsilon_{p}$ is the internal porosity, and $D_{s}$ is the surface diffusivity. In the current case of diluted systems, the following linear isotherm is used:

$$
q_{p}^{*}=a c_{p}
$$

In the above equation, $a$ denotes the Henry's coefficient. In order to simplify the notations and reduce the number of variables, the following dimensionless variables are introduced:

$$
\begin{aligned}
& C=\frac{c}{c_{\mathrm{inj}}}, \quad C_{p}=\frac{c_{p}}{c_{\mathrm{inj}}}, \quad \tau=\frac{u t}{L}, \quad x=\frac{z}{L}, \quad \psi=\frac{\rho}{R}, \quad r=\frac{r_{p}}{R_{P}}, \\
& P e_{z}=\frac{L u}{D_{z}}, \quad P e_{\rho}=\frac{R^{2} u}{D_{\rho} L}, \quad B i=\frac{k_{\mathrm{ext}} R_{p}}{D_{\mathrm{eff}}}, \quad \eta=\frac{D_{\mathrm{eff}} L}{u R_{p}^{2}}, \quad \xi=3 B i \eta F=\frac{3 L F k_{\mathrm{ext}}}{u R_{p}} .
\end{aligned}
$$


Here, $D_{\text {eff }}=\epsilon_{p} D_{p}+a\left(1-\epsilon_{p}\right) D_{s}$. After using the above dimensionless variables, Eqs. (1) and (2) take the forms

$$
\begin{aligned}
\frac{\partial C}{\partial \tau}+\frac{\partial C}{\partial x} & =\frac{1}{P e_{z}} \frac{\partial^{2} C}{\partial x^{2}}+\frac{1}{P e_{\rho}}\left(\frac{\partial^{2} C}{\partial \psi^{2}}+\frac{1}{\psi} \frac{\partial C}{\partial \psi}\right)-\xi\left(C-C_{p}(r=1)\right), \\
a^{*} \frac{\partial C_{p}}{\partial \tau} & =\frac{\eta}{r^{2}} \frac{\partial}{\partial r}\left(r^{2} \frac{\partial C_{p}}{\partial r}\right)
\end{aligned}
$$

where

$$
a^{*}=\epsilon_{p}+a\left(1-\epsilon_{p}\right) .
$$

The initial condition for Eq. (5), considering an initially regenerated column, is given as

$$
C(\psi, x, \tau=0)=0, \quad 0<x<1, \quad 0 \leq \psi \leq 1
$$

33 The initial condition corresponding to Eq. (6) is given as

$$
C_{p}(r, \psi, x, \tau=0)=0, \quad 0<x<1, \quad 0 \leq r \leq 1, \quad 0 \leq \psi \leq 1 .
$$

The Eq. (5) is subjected to the following boundary conditions (BCs) along the radial coordinate of the column:

$$
\frac{\partial C(\psi=0, x, \tau)}{\partial \psi}=0, \quad \frac{\partial C(\psi=1, x, \tau)}{\partial \psi}=0 .
$$

These BCs describe the symmetry of the radial profile and the impermeability of the column wall, respectively. Moreover, Eq. (6) is subjected to the following BCs:

$$
\frac{\partial C_{p}(r=0, \psi, x, \tau)}{\partial r}=0, \quad \frac{\partial C_{p}(r=1, \psi, x, \tau)}{\partial r}=B i\left(C-\left.C_{p}\right|_{r=1}\right) .
$$


The second BC in Eq. (11) at $r=1$ quantifies the temporal change of the average loading of the particles and describes a connection between Eqs. (5) and (6).

Two different types of boundary conditions (BCs) are considered for Eq. (5) at the column inlet and outlet. Moreover, the sample injection is either considered through an inner cylindrical core or an outer annular ring.

Case 1: Rectangular concentration pulse injected as Dirichlet inlet BC:

The left $\mathrm{BC}$ for sample injection through inner cylindrical region is given as

$$
C(\psi, x=0, \tau)=\left\{\begin{array}{cc}
1, & \text { if } 0 \leq \psi \leq \bar{\psi} \text { and } 0 \leq \tau \leq \tau_{\mathrm{inj}} \\
0, & \text { if } \bar{\psi}<\psi \leq 1 \text { or } \tau>\tau_{\mathrm{inj}}
\end{array}\right.
$$

while, the left BC for outer annular ring injection is described as

$$
C(\psi, x=0, \tau)=\left\{\begin{array}{cc}
1, & \text { if } \bar{\psi}<\psi \leq 1 \text { and } 0 \leq \tau \leq \tau_{\text {inj }} \\
0, & \text { if } 0 \leq \psi \leq \bar{\psi} \text { or } \tau>\tau_{\text {inj }}
\end{array}\right.
$$

Here,

$$
\bar{\psi}=\bar{\rho} / R \text {. }
$$

For injecting over the entire inlet cross-section of the column, either $\bar{\psi}=1$ in Eq. (12) or $\bar{\psi}=0$ in Eq. (13).

At the right end of hypothetically infinite length column $(x=\infty)$, the following outflow Neumann BC is considered:

$$
\left.\frac{\partial C}{\partial x}\right|_{x=\infty}=0 .
$$

\section{Case 2: Rectangular concentration pulse injected as Danckwerts inlet BC:}

For the inner cylindrical zone injection the corresponding left $\mathrm{BC}$ is given as

$$
C(\psi, x=0, \tau)-\frac{1}{P e_{z}} \frac{\partial C(\psi, x=0, \tau)}{\partial x}=\left\{\begin{array}{lc}
1, & \text { if } 0 \leq \psi \leq \bar{\psi} \text { and } 0 \leq \tau \leq \tau_{\text {inj }} \\
0, & \bar{\psi}<\psi \leq 1 \text { or } \tau>\tau_{\text {inj }}
\end{array}\right.
$$


while, for injection at outer annular ring, it is expressed as

$$
C(\psi, x=0, \tau)-\frac{1}{P e_{z}} \frac{\partial C(\psi, x=0, \tau)}{\partial x}=\left\{\begin{array}{lc}
1, & \text { if } \tilde{\psi}<\psi \leq 1 \text { and } 0 \leq \tau \leq \tau_{\mathrm{inj}} \\
0, & 0 \leq \psi \leq \tilde{\psi} \text { or } \tau>\tau_{\mathrm{inj}}
\end{array}\right.
$$

The following zero Neumann BC is applied at the right end of a finite length column:

$$
\left.\frac{\partial C}{\partial x}\right|_{x=1}=0
$$

\section{Derivation of analytical solutions}

The Eqs. (5) and (6) of the model together with the associated initial and boundary conditions are solved analytically by first applying the finite Hankel transform and then the Laplace transform.

The Hankel-transform is an integral transform, also known as the Fourier-Bessel transform, that expresses a given function as the weighted sum of an infinite number of Bessel functions of the first kind. The Hankel-transforms for annularly symmetric functions are the Fouriertransforms in two dimensions. They are used to determine the solutions of boundary value problems in cylindrical coordinates by eliminating the radial coordinate. The zeroth-order finite Hankel-transform $C_{H}\left(\lambda_{n}, x, \tau\right)$ of $C(\psi, x, \tau)$ is defined as $[15,16,41-43]$

$$
C_{H}\left(\lambda_{n}, x, \tau\right)=H[C(\psi, x, \tau)]=\int_{0}^{1} C(\psi, x, t) J_{0}\left(\lambda_{n} \psi\right) \rho d \psi
$$

where, $\lambda_{n}$ is the finite Hankel-transform parameter which is given in term of the transcendental equation as $\frac{d J_{0}\left(\lambda_{n}\right)}{d \psi}=-J_{1}\left(\lambda_{n}\right)=0$. Here, $J_{0}($.$) and J_{1}($.$) are the zeroth and first$ order Bessel functions of first kind, respectively. The inverse Hankel-transform is expressed as

$$
C(\psi, z, \tau)=2 c_{H}\left(\lambda_{n}=0, x, \tau\right)+2 \sum_{n=1}^{\infty} C_{H}\left(\lambda_{n}, x, \tau\right) \frac{J_{0}\left(\lambda_{n} \psi\right)}{\left|J_{0}\left(\lambda_{n}\right)\right|^{2}}
$$


The Hankel transformation of Eq. (5) with respect to coordinate $\psi$ gives

$$
\frac{\partial C_{H}}{\partial \tau}+\frac{\partial C_{H}}{\partial x}=\frac{1}{P e_{z}} \frac{\partial^{2} C_{H}}{\partial x^{2}}-\frac{\lambda_{n}^{2}}{P e_{\rho}} C_{H}-\xi\left(C_{H}-\left.C_{p H}\right|_{r=1}\right) .
$$

Here, $C_{H}\left(\lambda_{n}, x, \tau\right)$ and $C_{p H}\left(\lambda_{n}, x, \tau\right)$ are the zeroth-order finite Hankel transforms of $C(\psi, x, \tau)$ and $C_{p}(r, \psi, x, \tau)$, respectively.

The Laplace-transform is a widely used integral transform in mathematics and engineering. It is used to transform a function of time into a function of complex frequency. The inverse Laplace-transform on the other hand takes a function from the complex frequency domain into a function defined in the time domain. The Laplace-transform generally gives a function as a superposition of moments which is one of the key reasons for using this technique in the present work. The Laplace transformation of Hankel transformed function $C_{H}$ is defined as [43]

$$
\bar{C}_{H}\left(\lambda_{n}, x, s\right)=\int_{0}^{\infty} e^{-s t} C_{H}\left(\lambda_{n}, x, \tau\right) d t, \quad t \geq 0 .
$$

After applying the Laplace transformation on Eq. (21) with respect to $\tau$ and assuming that the initial concentration is zero, we get

$$
\frac{1}{P e_{z}} \frac{\partial^{2} \bar{C}_{H}}{\partial x^{2}}-\frac{\partial \bar{C}_{H}}{\partial x}-\left(s+\frac{\lambda_{n}^{2}}{P e_{\rho}}\right) \bar{C}_{H}-\xi\left(\bar{C}_{H}-\left.\bar{C}_{p H}\right|_{r=1}\right)=0
$$

Here, $\bar{C}_{H}$ denotes the Hankel and Laplace transformed concentration. After rephrasing Eq. (6), we obtain

$$
a^{*} \frac{\partial}{\partial \tau}\left(r C_{p}\right)-\eta \frac{\partial^{2}}{\partial r^{2}}\left(r C_{p}\right)=0
$$

After applying the Hankel and Laplace transformations on Eq. (24), we get

$$
\frac{d^{2}}{d r^{2}}\left(r \bar{C}_{p H}\right)-\alpha(s) r \bar{C}_{p H}=0
$$


where, $\alpha(s)=\frac{a^{*} s}{\eta}$. Here, $A$ and $B$ are constants of integration which are determined through boundary conditions in Eq. (11). Thus, we obtain

$$
A=\frac{B i \bar{C}_{H} / 2 \sinh (\sqrt{\alpha(s)})}{(B i-1)+\sqrt{\alpha(s)} \operatorname{coth}(\sqrt{\alpha(s)})}, \quad B=-\frac{B i \bar{C}_{H} / 2 \sinh (\sqrt{\alpha(s)})}{(B i-1)+\sqrt{\alpha(s)} \operatorname{coth}(\sqrt{\alpha(s)})} .
$$

Therefore, the solution in Eq. (26) at $r=1$, takes the form

$$
\left.\bar{C}_{p H}\right|_{r=1}=\bar{C}_{H} f(s), \quad f(s)=\frac{B i}{(B i-1)+\sqrt{\alpha(s)} \operatorname{coth}(\sqrt{\alpha(s)})}
$$

After introducing Eq. (28) in Eq. (23), we get the following ordinary differential equation:

$$
\frac{d^{2} \bar{C}_{H}}{d x^{2}}-P e_{z} \frac{d \bar{C}_{H}}{d x}-P e_{z} \phi\left(s, \lambda_{n}\right) \bar{C}_{H}=0
$$

where

$$
\phi\left(s, \lambda_{n}\right)=s+\frac{\lambda_{n}^{2}}{P e_{\rho}}+\xi(1-f(s)) .
$$

190 The solution of Eq. (29) is given as

$$
\bar{C}_{p H}\left(\lambda_{n}, x, \tau\right)=A_{0} e^{m_{1} x}+B_{0} e^{m_{2} x},
$$

where

$$
m_{1,2}=\frac{P e_{z}}{2}\left(1 \pm \sqrt{1+\frac{4 \phi\left(s, \lambda_{n}\right)}{P e_{z}}}\right)
$$


197

The positive sign (upper case) in Eq. (32) is selected for calculating $m_{1}$ and the negative sign is used for calculating $m_{2}$. The integration constants $A_{0}$ and $B_{0}$ have to be determined from the given axial BCs as given below.

Case 1: Rectangular concentration pulse injection as Dirichlet inlet BC:

The Hankel-transform of Eqs. (12) (or (13)) and (15) are

$$
C_{H}\left(\lambda_{n}, x=0, \tau\right)=\left\{\begin{array}{lc}
F\left(\lambda_{n}\right), & \text { if } 0 \leq \tau \leq \tau_{\text {inj }} \\
0, & \text { if } \tau>\tau_{\text {inj }}
\end{array}\right.
$$

$$
\left.\frac{\partial C_{H}\left(\lambda_{n}, x, \tau\right)}{\partial x}\right|_{x=\infty}=0
$$

For inner cylindrical core injection, $F\left(\lambda_{n}\right)$ is

$$
F\left(\lambda_{n}\right)=\left\{\begin{array}{lr}
\frac{\tilde{\psi}^{2}}{2}, & \text { if } \lambda_{n}=0 \\
\frac{\tilde{\psi}}{\lambda_{n}} J_{1}\left(\lambda_{n} \tilde{\psi}\right), & \text { if } \lambda_{n} \neq 0
\end{array}\right.
$$

and for outer annular ring injection, it is given as

$$
F\left(\lambda_{n}\right)= \begin{cases}\left(\frac{1}{2}-\frac{\tilde{\psi}^{2}}{2}\right), & \text { if } \lambda_{n}=0 \\ -\frac{\tilde{\psi}}{\lambda_{n}} J_{1}\left(\lambda_{n} \tilde{\psi}\right), & \text { if } \lambda_{n} \neq 0 .\end{cases}
$$

Using the Laplace-transform on BCs in Eqs. (33) and (34), we obtain

$$
\bar{C}_{H}\left(\lambda_{n}, x=0, s\right)=\frac{F\left(\lambda_{n}\right)}{s}\left(1-e^{-s \tau_{\mathrm{inj}}}\right),\left.\quad \frac{\partial \bar{C}_{H}}{\partial x}\right|_{x=\infty}=0 .
$$

On using Eq. (37) in Eq. (31), we get

$$
A_{0}=0, \quad B_{0}=\frac{\left(1-e^{-s \tau_{\mathrm{inj}}}\right)}{s} F\left(\lambda_{n}\right) .
$$

Therefore, the solution in Eq. (31) becomes:

$$
\bar{C}_{H}\left(\lambda_{n}, x, s\right)=\frac{\left(1-e^{-s \tau_{\mathrm{inj}}}\right)}{s} F\left(\lambda_{n}\right) e^{m_{2} x},
$$


where, $m_{2}$ is given by Eq. (32) for the lower minus sign. In the current scenario, no analytical Laplace inversion is possible. For that reason, the numerical Laplace inversion will be applied to get semi-analytical solutions in the actual time domain [38]. In this article the well established numerical inversion method for Laplace transforms, based on a Fourier series expansion developed by Durbin [44], is applied. Generally, the discretization and truncation errors of this method depend on the free parameters involved in the technique. However, there are several procedures available which can be used in the method to reduce the discretization error, to accelerate the convergence of the Fourier series and to compute good choice of the free parameters [45]. Suitable for a given problem, the inversion method allows the adequate application of these procedures. Therefore, in a wide range of applications a high accuracy can be achieved rapidly evaluating only a few function evaluations of the Laplace transform. In this manuscript, the required results were obtained in a less than a minute time on a PC.

\section{Case 2: Rectangular concentration pulse injection as Danckwerts inlet BC:}

The Hankel-transform of Eqs. (16) (or (17)) and (18) are

$$
C_{H}\left(\lambda_{n}, x=0, \tau\right)-\frac{1}{P e_{z}} \frac{\partial C_{H}\left(\lambda_{n}, x=0, \tau\right)}{\partial x}=\left\{\begin{array}{lc}
F\left(\lambda_{n}\right), & \text { if } 0 \leq \tau \leq \tau_{\text {inj }} \\
0, & \text { if } \tau>\tau_{\text {inj }}
\end{array}\right.
$$

and

$$
\left.\frac{\partial C_{H}\left(\lambda_{n}, x, \tau\right)}{\partial x}\right|_{x=1}=0
$$

Here, $F\left(\lambda_{n}\right)$ is given by Eq. (35) for inner cylindrical core injection and by Eq. (36) for outer annular region injection.

Application of Laplace-transform on the above BCs gives

$$
\bar{C}_{H}\left(\lambda_{n}, x=0, s\right)-\frac{1}{P e_{z}} \frac{\partial \bar{C}_{H}\left(\lambda_{n}, x=0, s\right)}{\partial x}=\frac{F\left(\lambda_{n}\right)}{s}\left(1-e^{-s \tau_{\mathrm{inj}}}\right)
$$

and

$$
\left.\frac{\partial \bar{C}_{H}}{\partial x}\right|_{x=1}=0
$$


By using Eqs. (42) and (43) in Eq. (31), we get the values of $A_{0}$ and $B_{0}$ as

$$
A_{0}=\frac{m_{2} e^{m_{2}}\left(\frac{F\left(\lambda_{n}\right)}{s}\left(1-e^{-s \tau_{\mathrm{inj}}}\right)\right)}{m_{2} e^{m_{2}}\left(1-\frac{m_{1}}{P e_{z}}\right)-m_{1} e^{m_{1}}\left(1-\frac{m_{2}}{P e_{z}}\right)},
$$

and

$$
B_{0}=-\frac{m_{2} e^{m_{2}}\left(\frac{F\left(\lambda_{n}\right)}{s}\left(1-e^{-s \tau_{\mathrm{inj}}}\right)\right)}{m_{2} e^{m_{2}}\left(1-\frac{m_{1}}{P e_{z}}\right)-m_{1} e^{m_{1}}\left(1-\frac{m_{2}}{P e_{z}}\right)} .
$$

Thus, the solution in Eq. (31) becomes

$$
\bar{C}_{H}\left(\lambda_{n}, x, s\right)=\frac{\left[m_{2} e^{m 2+m_{1} x}-m_{1} e^{m 1+m_{2} x}\right]\left[\frac{F\left(\lambda_{n}\right)}{s}\left(1-e^{-s \tau_{\mathrm{inj}}}\right)\right]}{m_{2} e^{m_{2}}\left(1-\frac{m_{1}}{P e_{z}}\right)-m_{1} e^{m_{1}}\left(1-\frac{m_{2}}{P e_{z}}\right)} .
$$

Here, the value of $m_{1}$ and $m_{2}$ are given by Eq. (32). Once again analytical Laplace inversion is not possible. Thus, the numerical Laplace inversion will be applied to get semi-analytical solutions in the actual time domain $[38,44]$.

\section{Moment Analysis}

Moment analysis is a well known useful technique for collecting relevant information about the retention equilibrium and mass transfer kinetics in a column. A set of statistical temporal moments can be used to describe the appearance of elution profile. For instance, the appropriate forms of first, second, third and fourth moments can describe the mean, spread, skewness, and kurtosis of the elution profiles, respectively. The experimental values measured for these moments can be compared with their theoretical expressions to estimate mass transfer coefficients.

The normalized $i$-th moment averaged over the radial coordinate $(\psi)$ of the band profile at any position in the column can be obtained through the following expression

$$
\mu_{0, \mathrm{av}}=\int_{0}^{\infty} C_{\mathrm{av}}(x, \tau) d \tau, \quad \mu_{i, \mathrm{av}}=\frac{\int_{0}^{\infty} C_{\mathrm{av}}(x, \tau) \tau^{i} d \tau}{\mu_{0, \mathrm{av}}}, \quad i=1,2,3, \cdots
$$


where

$$
C_{\mathrm{av}}(x, \tau)=2 \int_{0}^{1} C(\psi, x, \tau) \psi d \psi .
$$

Due to its moment generating property, the Laplace transformation can be used to obtain analytical expressions for the moments. Temporal moments are derived analytically as functions of radial coordinate $\psi$ at the outlet of the column $(x=1)$. The following relation is utilized to obtain analytical temporal moments from the Hankel and Laplace transformed concentration $\bar{C}_{H}$ in Eq. (39) or (46)

$$
\mu_{i, H}=(-1)^{i} \lim _{s \rightarrow 0} \frac{d^{i}\left(\bar{C}_{H}\left(\lambda_{n}, x, s\right)\right)}{d s^{i}}, \quad i=0,1,2, \cdots
$$

The true moments $\mu_{i}(\psi)$ are obtained from Eq. (20) by taking the $i$-th moment of concentration on the both sides of that equation. Thus, on multiplying both sides of Eq. (20) with $\tau^{i}$ and integrating over $\tau$ from 0 to $\infty$, we get

$$
\mu_{i}(\psi)=2 \mu_{i, H}\left(\lambda_{n}=0\right)+2 \sum_{n=1}^{\infty} \mu_{i, H}\left(\lambda_{n}\right) \frac{J_{0}\left(\lambda_{n} \psi\right)}{\left|J_{0}\left(\lambda_{n}\right)\right|^{2}}
$$

Further, the averaged non-normalized temporal moments $M_{i \text {,av }}$ are determined as

$$
M_{i, \mathrm{av}}=2 \int_{0}^{1} \mu_{i}(\psi) \psi d \psi, \quad i=0,1,2, \cdots
$$

Lastly, the normalized averaged temporal moments, defined in Eq. (47) and frequently used in chemical engineering [2], are given as

$$
\mu_{i, \mathrm{av}}=\frac{M_{i, \mathrm{av}}}{\mu_{0, \mathrm{av}}}, \quad \mu_{0, \mathrm{av}}=M_{0, \mathrm{av}}, \quad i=1,2,3, \cdots
$$


The above temporal moments $\mu_{i \text {,av }}$ up to fourth order are obtained to interpret the behavior of a solute moving through the column. The first three central moments can be obtained as $[2]$

$$
\begin{aligned}
& \mu_{2, \mathrm{av}}^{\prime}=\mu_{2, \mathrm{av}}-\mu_{1, \mathrm{av}}^{2}, \\
& \mu_{3, \mathrm{av}}^{\prime}=\mu_{3, \mathrm{av}}-3 \mu_{1, \mathrm{av}} \mu_{2, \mathrm{av}}+2 \mu_{1, \mathrm{av}}^{3}, \\
& \mu_{4, \mathrm{av}}^{\prime}=\mu_{4, \mathrm{av}}-4 \mu_{1, \mathrm{av}} \mu_{3, \mathrm{av}}+6 \mu_{1, \mathrm{av}}^{2} \mu_{2, \mathrm{av}}-3 \mu_{1, \mathrm{av}}^{4} .
\end{aligned}
$$

The corresponding numerical $i$-th moment of the band profile at the outlet of the column of length $x=1$ is obtained as

$$
\mu_{i, \mathrm{av}}=\frac{\int_{0}^{\infty} C_{\mathrm{av}}(x=1, \tau) \tau^{i} d \tau}{\mu_{0, \mathrm{av}}}, \quad i=2,3,4, \cdots
$$

where, $\mu_{0, \text { av }}$ for $x=1$ is given by Eq. (47). The trapezoidal rule is utilized to approximate the integrals appearing in Eqs. (47) and (56).

The analytical moments of 2D-GRM for the considered two sets of boundary conditions are presented in Appendix A.

\section{Numerical test problems}

The analytical solutions and moments derived in the previous sections are validated by considering several test problems. The high resolution finite volume scheme (HR-FVS) of Koren is applied to numerically approximate the model equations for verifying the correctness of analytical results $[39,40]$. In these test problems, the effects of model parameters $P e_{z}, P e_{\rho}, B i$ and $\eta$ characterizing the axial dispersion, radial dispersion, film mass transfer resistance, and intraparticle diffusion resistance, are analyzed on the elution profiles and moments. The parameters used in the test problems are given in Table 1. These parameters are taken from the ranges typically encountered in HPLC applications. Hereby the considered axial Peclet number of 600 is connected by a factor of 2 with the traditional 
number of theoretical plates. Thus, a low efficient HPLC column is described in our calculations. More difficult is indeed the quantification of the radial Peclet number. For the relative small particles used in chromatographic columns there are no reliable measurements or correlations for these numbers available. To capture the order of magnitude, we evaluated the following ratio of the two Peclet numbers based on their definitions in Eq. $(4)$

$$
\frac{P e_{z}}{P e_{\rho}}=\frac{L^{2}}{R^{2}} \frac{D_{\rho}}{D_{z}} .
$$

Two trends that can be used to estimate this ratio: (a) columns have typically much larger $L$ (length) values than $R$ (radius) values, (b) due to the missing radial convection, $D_{\rho}$ is smaller than $D_{z}$. Hereby, the first contribution is more pronounced, leading to the larger axial Peclet number than the corresponding radial Peclet number. Based on this argument, we considered the value of 15 for the radial Peclet number, which was mostly applied in our calculations, as reasonable.

\subsection{Effect of boundary conditions}

Figures 2 and 3 show the 3D and 1D plots of the concentration profiles for Danckwerts BCs using the solution Eq. (46). In these case studies, the sample was either injected in the inner cylindrical region or outer annular region at $x=0$. The radius of the inner cylindrical core $\bar{\rho}$ is chosen such that the areas of both inner and outer regions are the same. Thus, for the considered column of radius $R=0.2$, the inside annular region radius comes out to be $\bar{\rho}=0.1414$. The parameters used in the test problems are given in Table 1.

Figure 2 shows the plots of concentration profiles for inner zone injection. For the considered radial Peclet number of 15 , the transport rate along the radial coordinate is quite small. Thus, a variation in the contraction profile is still visible at the column outlet in Figure 2(a) along the radial coordinate. The plots of the concentration profiles in the center of the column in Figure 2(b) shows a good agreement between the analytical and numerical results. 
Figure 3 shows the result for injection through outer zone. The same parameters are used as in Figure 2. Now, still at the column outlet a higher concentration can be seen in the outer region as compared to the inner cylindrical region due to slow radial dispersion. Once again, a good agreement can be observed between the analytical and numerical results. Figures 2c and 3c further show the outlet concentration profiles averaged over the whole radius. These plots show a strong similarity for the considered two types of injections. This indicates that for the parameters considered there is no clear preference for one or the other injection type. In predicting the radially averaged elution profiles the $1 \mathrm{D}$ and $2 \mathrm{D}$ models provide very similar results. The 1D solutions in Figures $2 \mathrm{c}$ and $3 \mathrm{c}$ were available from our previous article [46] by considering $c_{\text {inj }}=0.5$, as the inlet cross-sectional area of the column has been divided into equal areas of inner and outer injection zones. As stated above, our goal here is essentially to provide challenging test scenarios for the mathematical solution of the 2D model capable to predict the dynamics of radial gradients.

Figures $4 \mathrm{a}$ and $4 \mathrm{~b}$ display the 3D plots of outlet concentration profiles for injection through outer zone using smaller and larger radial Peclet numbers, i.e. $P e_{\rho}=1.5$ and $P e_{\rho}=150$. Once again the solution in Eq. (46) for Danckwerts BCs is used. The other parameters are exactly the same as given in Table 1 and used in Figures 2 and 3. These plots describe chromatograms for columns of smaller and larger diameters expressed by smaller and larger radial Peclet numbers, respectively (c.f. Eq. (4)). It can be seen from Figure $4 b$ that value of the liquid concentration reduces at the center of the column for large radial Peclet number (here 150). Slow radial dispersion (large radial Peclet number) clearly reduces the rate of eliminating gradients caused by the injection. Figure 4c shows for the outlet concentrations in the column center that the difference between the solutions of $1 \mathrm{D}$ and 2D models is more pronounced in the case of larger radial Peclet numbers (i.e. larger diameter column) as compared to the case of smaller radial Peclet numbers (i.e. smaller diameter column). Moreover, the plots in Figure 4d show that radial profiles at the mean retention time are quite different in the two cases. Only for sufficiently small radial Peclet numbers the $1 \mathrm{D}$ model is sufficient. These results illustrate that the considered 2D model correctly describe the evolution of radial mass transfer and can provide more detailed insight into 
the column dynamics.

Figure 5 gives a comparison of analytical solutions for the Dirichlet and Danckwerts BCs. The results of both boundary conditions are the same for large $P e_{z}$. However, differences in the solutions can be observed for small values of $P e_{z}$. Thus, for small $P e_{z}$ (or large $D_{z}$ ) the more realistic Danckwerts BCs should be used. The Danckwerts boundary conditions have capability to quantify the unavoidable back mixing at the column inlet and predict broader profiles.

\subsection{Effects of Kinetic Parameters}

Figure 6 shows the effects of $P e_{z}, P e_{\rho}, B i$ and $\eta$ on the concentration profiles. It is evident from the Figure 6(a) that if the axial dispersion is more rate limiting, the peak becomes wider and its height decreases. In other words, the column efficiency reduces on decreasing the value of $P e_{z}$.

Figure 6(b) displays the plots of radial concentration profiles at the middle of the column ( $x=0.5)$ using different values of $P e_{\rho}$ and keeping $P e_{z}=600$ fixed. It can be observed that the imposed step profiles deteriorate faster for small $P e_{\rho}$ (or larger radial dispersion coefficient $D_{\rho}$ ). The limiting case $P e_{\rho}=15$ corresponds to the elimination of injection profiles.

The effect of the model parameters $B_{i}$ and $\eta$, which contain the mass transfer coefficient $K_{\text {ext }}$ and intraparticle diffusivity $D_{\text {eff }}$, are shown in Figures 6(c) and (d). For small values of $B i$ and $\eta$, the mass transfer and diffusion rates are relatively slow. Thus, the peak are tailed and broadened.

\subsection{Discussion on analytical moments}

The plots of dimensionless moments show the effects of the radial and axial dispersion coefficients on the concentration profile using inner zone injection. The Dirichlet boundary conditions were considered, as Danckwerts boundary conditions give the same results. Figure 7 gives the plots of averaged moments. These moments have good agreement with the results for concentration profiles. There are no effects of the radial dispersion coefficient 
on the averaged moments but its effects can be seen in the local moments displayed in Figure 8. Figure 7(a) shows that there is no effect of axial dispersion coefficient on the first moment as expected. The effect of $P e_{z}$ is clearly seen in the second, third and fourth moments. The plots in Figure 7 also show a comparison between analytically and numerically determined moments which are in good agreement with each other. Figure 8 displays the local moments plotted against radial coordinate $\psi$. Different values of $P e_{\rho}$ were assumed. Variations in the local moments can be seen for large $P e_{\rho}$ (or small radial dispersion coefficient $D_{\rho}$ ). The behaviors of these results are in good agreement with those presented in Figure 6(b).

\subsection{Matching of 2D-LKM and 2D-GRM kinetic parameters}

Figure 9 compares the results of 2D-GRM with those of simplified two-dimensional lumped kinetic model (2D-LKM). The results of 2D-LKM were obtained by choosing the values of its parameters according to the relations given in Eqs. (58) and (59) [23, 46]. Figure 9(a) shows the plots of zeroth moments with respect to $P e_{z}$. It can be observed that both models have the same zeroth moments for the same inlet conditions. The first moments of 2D-GRM and 2D-LKM were matched through the following relation (c.f. [46])

$$
a=a^{*},
$$

where, a denotes the Henry's constant in LKM model [23]. To match second moments, the following relations between the parameters of 2D-GRM and 2D-LKM were used (c.f. $[23,46])$

$$
a=a^{*}, \quad \kappa=\left[\frac{a^{*}}{1-\epsilon_{e}}\left(\frac{1}{15 \eta}+\frac{F}{\xi}\right)\right]^{-1},
$$

where, $\kappa$ denotes the dimensionless overall mass transfer coefficient in 2D-LKM [23]. Figures 9(b) and 9(c) verify that for these values of parameters, the first and second moments of 2D-GRM and 2D-LKM are identical. However, these relations do not guarantee the 
matching of high order moments. For these values of $a$ and $\kappa$ in 2D-LKM, the retention times times (first moments) and the variances (second moments) of the concentration profiles are the same as depicted in Figure 9b and 9c. The almost perfect matching of both bands is due to their almost perfect approximate Gaussian profiles, i.e. skewness is almost zero. Thus, even third and fourth moments of both models were found being very close to each others. However, for skewed profiles the third and high order moment of both models will not match perfectly with each other, as the matching of only first two moments is guaranteed through above relations. To match high order moments, we have to derive new relations for $\kappa$ by comparing the corresponding moments of both models. However, a particular value of $\kappa$ obtained by matching the corresponding high order moments (for example third moments) of both model will only guarantee the equivalence of those specific moments (i.e. third moments) and the remaining moments of second and high order will be different.

The results discussed here reveal that for linear isotherms there is significant potential to use simplified models with less parameters. If the parameters applied guarantee a matching of the first two moments, predicted elution profiles will be very similar for both types of models.

\section{Conclusion}

Analytical solutions and moments of a two-dimensional linear general rate model were derived to simulate a single-solute transport in the chromatographic columns of cylindrical geometry. The analytical solutions were derived by successively applying the finite Hankel and Laplace transformations. The solutions were derived for two sets of boundary conditions and considering injections through inner and outer zones of the column inlet cross section. The developed analytical solutions extend our previous analysis by incorporating the influences of mass transfer coefficient, intraparticle diffusion, and longitudinal and radial dispersion coefficients. The derived analytical solutions were compared for verification with the numerical solutions of a high resolution flux limiting finite volume scheme. Moreover, relations were derived among the kinetic parameters of 2D-LKM and 2D-GRM to 


$$
\mu_{2, H}=\left[\frac{\tau_{\mathrm{inj}}^{2}}{3}+\frac{\left(1+a^{*} F\right)}{w} \tau_{\mathrm{inj}}+\frac{\left(1+a^{*} F\right)^{2}}{w^{2}}+\frac{2\left(1+a^{*} F\right)^{2}}{P e_{z} w^{3}}+\frac{2 a^{*^{2}} F(B i+5)}{15 w \eta B i}\right] \mu_{0, H}
$$


Third moment: The third temporal moment is obtained as

$$
\begin{aligned}
\mu_{3, H}= & {\left[\frac{\tau_{\mathrm{inj}}^{3}}{4}+\frac{\left(1+a^{*} F\right)}{w} \tau_{\mathrm{inj}}^{2}+\left(\frac{3\left(1+a^{*} F\right)^{2}}{2 w^{2}}+\frac{a^{* 2} F(B i+5)}{5 w B i \eta}+\frac{3\left(1+a^{*} F\right)^{2}}{P e_{z} w^{3}}\right) \tau_{\text {inj }}\right.} \\
& +\frac{4 a^{*} F\left(\frac{35}{2}+7 B i+B i^{2}\right)}{105 w B i^{2} \eta^{2}}+\frac{12\left(1+a^{*} F\right)^{3}}{P e_{z}^{2} w^{5}}+\frac{4 a^{* 2} F\left(1+a^{*} F\right)(B i+5)}{5 P e_{z} w^{3} B i \eta} \\
& \left.+\frac{6\left(1+a^{*} F\right)^{3}}{P e_{z} w^{4}}+\frac{2 a^{* 2} F\left(1+a^{*} F\right)(B i+5)}{5 w^{2} B i \eta}+\frac{\left(1+a^{*} F\right)^{3}}{w^{3}}\right] \mu_{0, H} .
\end{aligned}
$$

424

Fourth moment: The fourth temporal moment is given as

$$
\begin{aligned}
\mu_{4, H}= & {\left[\frac{\tau_{\text {inj }}^{4}}{5}+\frac{\left(1+a^{*} F\right) \tau_{\text {inj }}^{3}}{w}+\left(\frac{4\left(1+a^{*} F\right)^{2}}{P e_{z} w^{3}}+\frac{4 a^{* 2} F(B i+5)}{15 w B i \eta}+\frac{2\left(1+a^{*} F\right)^{2}}{w^{2}}\right) \tau_{\text {inj }}^{2}\right.} \\
& +\left(\frac{2\left(1+a^{*} F\right)^{3}}{w^{3}}+\frac{8 a^{* 2} F\left(1+a^{*} F\right)(B i+5)}{5 P e_{z} w^{3} B i \eta}+\frac{8 a^{* 3} F\left(\frac{35}{2}+7 B i+B i^{2}\right)}{105 w B i^{2} \eta^{2}}\right. \\
& \left.+\frac{24\left(1+a^{*} F\right)^{3}}{P e_{z}^{2} w^{5}}+\frac{12\left(1+a^{*} F\right)^{3}}{P e_{z} w^{4}}+\frac{4 a^{* 2} F\left(1+a^{*} F\right)(B i+5)}{5 w^{2} B i \eta}\right) \tau_{\text {inj }} \\
& +\frac{32 a^{* 3} F\left(1+a^{*} F\right)\left(\frac{35}{2}+7 B i+B i^{2}\right)}{105 P e_{z} w^{3} B i^{2} \eta^{2}}+\frac{48 a^{* 2} F\left(1+a^{*} F\right)^{2}(B i+5)}{5 P e_{z}^{2} w^{5} B i \eta}+\frac{\left(1+a^{*} F\right)^{4}}{w^{4}} \\
& +\frac{8 a^{* 4} F^{2}(B i+5)^{2}}{75 P e_{z} w^{3} B i^{2} \eta^{2}}+\frac{24 a^{* 2} F\left(1+a^{*} F\right)^{2}(B i+5)}{5 P e_{z} w^{4} B i \eta}+\frac{4 a^{* 4} F^{2}(B i+5)^{2}}{75 w^{2} B i^{2} \eta^{2}} \\
& +\frac{120\left(1+a^{*} F\right)^{4}}{P e_{z} w^{7}}+\frac{8 a^{* 3} F\left(1+a^{*} F\right)\left(\frac{35}{2}+7 B i+B i^{2}\right)}{105 w^{2} B i^{2} \eta^{2}}+\frac{60\left(1+a^{*} F\right)^{4}}{P e_{z}^{2} w^{6}} \\
& \left.+\frac{12\left(1+a^{*} F\right)^{4}}{P e_{z} w^{5}}+\frac{4 a^{* 2} F\left(1+a^{*} F\right)^{2}(B i+5)}{5 w^{3} B i \eta}+\frac{8 a^{* 4} F\left(175+105 B i+27 B i^{2}\right)}{1575 w B i^{3} \eta^{3}}\right] \mu_{0, H} .
\end{aligned}
$$

\section{Case 2: Rectangular concentration pulse injection as Danckwert BC:}

In this case, the first four temporal moments are obtained from the Hankel and Laplace domains solutions in Eq. (46) using the moments generating relation in Eq. (49).

Zeroth moment: The zeroth moment is given as

$$
\mu_{0, H}=\frac{4 \tau_{\text {inj }} F\left(\lambda_{n}\right) e^{P e_{z}} w}{(w+1)^{2} e^{\frac{P e_{z}(w+1)}{2}}-(w-1)^{2} e^{-\frac{P e_{z}(w-1)}{2}}},
$$


where, $w$ ia given by Eq. (55). Let us define

$$
\begin{aligned}
& \beta_{1}=e^{\frac{P e_{z}(w+1)}{2}}, \quad \beta_{2}=e^{\frac{-P e_{z}(w-1)}{2}}, \quad \beta_{3}=4(w+1)+P e_{z}(w+1)^{2}, \\
& \beta_{4}=4(w-1)-P e_{z}(w-1)^{2}, \quad \beta_{5}=w+1, \quad \beta_{6}=w-1, \\
& \beta_{7}=(w+1)^{2} e^{\frac{P e_{z}(w+1)}{2}}-(w-1)^{2} e^{\frac{-P e_{z}(w-1)}{2}} .
\end{aligned}
$$

First Moment: The first moment for $i=1$ is given as

$$
\mu_{1, H}=\left[\frac{\tau_{\text {inj }}}{2}+\frac{\left(1+a^{*} F\right)}{P e_{z} w}\left(\frac{\beta_{3} \beta_{1}-\beta_{4} \beta_{2}}{\beta_{7}}-\frac{2}{w}\right)\right] \mu_{0, H} .
$$

Second Moment: The Second moment is expressed as

$$
\begin{aligned}
\mu_{2, H}= & {\left[\frac{\tau_{\text {inj }}^{2}}{3}+\left[\frac{\left(1+a^{*} F\right)}{P e_{z} w}\left(\frac{\beta_{3} \beta_{1}-\beta_{4} \beta_{2}}{\beta_{7}}-\frac{2}{w}\right)\right] \tau_{\text {inj }}+\frac{2\left(1+a^{*} F\right)^{2}}{P e_{z}^{2} w^{2}}\left(\frac{\beta_{3} \beta_{1}-\beta_{4} \beta_{2}}{\beta_{7}}\right)^{2}\right.} \\
& -\frac{4\left(1+a^{*} F\right)^{2}}{P e_{z}^{2} w^{3}}\left(\frac{\beta_{3} \beta_{1}-\beta_{4} \beta_{2}}{\beta_{7}}\right)+\frac{2\left(1+a^{*} F\right)\left(\beta_{5}^{2} \beta_{1}+\beta_{6}^{2} \beta_{2}\right)}{P e_{z} w^{3} \beta_{7}}+\frac{8\left(1+a^{*} F\right)^{2}\left(\beta_{1}-\beta_{2}\right)}{P e_{z}^{2} w^{2} \beta_{7}} \\
& +\frac{2}{15}\left(\frac{\left(\beta_{3} \beta_{1}-\beta_{4} \beta_{2}\right)-\left(\beta_{5}^{2} \beta_{1}+\beta_{6}^{2} \beta_{2}\right)}{P e_{z} w B i \eta \beta_{7}}\right) a^{* 2} F(B i+5)+\frac{\left(1+a^{*} F\right)^{2}}{w^{2}} \\
& -\frac{8\left(1+a^{*} F\right)^{2}\left(\beta_{6} \beta_{2}+\beta_{5} \beta_{1}\right)}{P e_{z} w^{2} \beta_{7}}+\frac{8\left(1+a^{*} F\right)^{2}\left(\beta_{6} \beta_{2}-\beta_{5} \beta_{1}\right)}{P e_{z}^{2} w^{3} \beta_{7}} \\
& \left.+\frac{4}{P e_{z} w^{2}}\left(\frac{a^{* 2} F(B i+5)}{15 B i \eta}-\frac{\left(1+a^{*} F\right)^{2}}{P e_{z} w^{2}}\right)+\frac{8 a^{* 2} F(B i+5)\left(\beta_{5} \beta_{1}-\beta_{6} \beta_{2}\right)}{15 P e_{z} w B i \eta \beta_{7}}\right] \mu_{0, H} .
\end{aligned}
$$

Let us define

$$
\begin{aligned}
\beta_{8} & =\frac{\beta_{3} \beta_{1}-\beta_{4} \beta_{2}}{P e_{z} w \beta_{7}}\left(1+a^{*} F\right), \quad \beta_{9}=\frac{a^{* 2} F(B i+5)}{5 P e_{z} w^{2} B i \eta}-\frac{3\left(1+a^{*} F\right)^{2}}{P e_{z}^{2} w^{4}}, \quad \beta_{10}=\frac{\beta_{5}^{2} \beta_{1}+\beta_{6}^{2} \beta_{2}}{P e_{z} w^{3} \beta_{7}}, \\
\beta_{11} & =\frac{\beta_{6} \beta_{2}+\beta_{5} \beta_{1}}{P e_{z} w^{2} \beta_{7}}, \beta_{12}=\frac{\beta_{1}-\beta_{2}}{P e_{z}^{2} w^{2} \beta_{7}}, \quad \beta_{13}=\frac{\beta_{6} \beta_{2}-\beta_{5} \beta_{1}}{P e_{z} w^{2} \beta_{7}}, \\
\beta_{14} & =\left[\frac{\left(\beta_{3} \beta_{1}-\beta_{4} \beta_{2}\right)-\left(\beta_{5}^{2} \beta_{1}-\beta_{6}^{2} \beta_{2}\right)}{5 P e_{z} w B i \eta \beta_{7}}\right] a^{* 2} F, \quad \beta_{15}=\frac{a^{* 2} F\left(\beta_{5} \beta_{1}-\beta_{6} \beta_{2}\right)}{5 P e_{z} w B i \eta \beta_{7}}, \\
\beta_{16} & =\frac{(1+a * F)\left(\beta_{1}+\beta_{2}\right)}{P e_{z}^{2} w^{2} \beta_{7}} .
\end{aligned}
$$

Third Moment: The third moment is given as 


$$
\begin{aligned}
\mu_{3, H}= & {\left[\frac{\tau_{\text {inj }}^{3}}{4}-\left(\beta_{8}-\frac{2}{P e_{z} w^{2}}\right) \tau_{\text {inj }}^{2}-\left(\frac{6\left(1+a^{*} F\right)}{P e_{z} w} \beta_{8}+3\left(1+a^{*} F\right) \beta_{10}+(B i+5) \beta_{14}\right.\right.} \\
& \left.+4(B i+5) \beta_{15}+4 \beta_{9}+3 \beta_{8}^{2}-\left(1+a^{*} F\right)^{2}\left(12 \beta_{11}-12 \beta_{12}-\frac{3}{2 w^{2}}-\frac{12 \beta_{13}}{P e_{z}}\right)\right) \tau_{\text {inj }} \\
& +\left(6 \beta_{8}-\frac{6\left(1+a^{*} F\right)}{P e_{z} w^{2}}\right)\left(2\left(1+a^{*} F\right) \beta_{10}-\left(1+a^{*} F\right)^{2}\left(8 \beta_{11}-8 \beta_{12}-\frac{1}{w^{2}}-\frac{8 \beta_{13}}{P e_{z} w}\right)\right. \\
& \left.-\frac{2 a^{* 2} F P e_{z} w^{2}(B i+5) \beta_{10}}{15 B i \eta}+\frac{8}{3}(B i+5) \beta_{15}\right)+\left(1+a^{*} F\right)\left(\frac{16(B i+5) \beta_{15}}{P e_{z} w^{2}}-\frac{4 a^{* 2} F \beta_{10}}{5 B i \eta}\right. \\
& +\frac{24 \beta_{16}}{w}+\frac{2 a^{* 2} F(B i+5) P e_{z} \beta_{13}}{5 B i \eta}+\frac{16 a^{* 2} F(B i+5) P e_{z} \beta_{11}}{5 B i \eta}-\frac{8 a^{* 2} F(B i+5)}{P e_{z}^{2} w^{4}} \\
& \left.+\frac{12 \beta_{8}^{2}}{P e_{z} w^{2}}\right)+\left(1+a^{*} F\right)^{3}\left(12 \beta_{13}+P e_{z} \beta_{10}+\frac{12 \beta_{10}}{P e_{z} w^{2}}+\frac{48 \beta_{13}}{P e_{z}^{2} w^{3}}+\frac{6}{P e_{z} w^{4}}-\frac{48 \beta_{11}}{P e_{z} w^{2}}\right. \\
& \left.+\frac{48 \beta_{12}}{P e_{z} w^{2}}+\frac{24}{P e_{z}^{3} w^{6}}\right)+\left(\frac{35}{2}+7 B i+B i^{2}\right)\left(\frac{16 a^{*} F \beta_{15}}{21 B i \eta}+\frac{4 a^{* 3} F \beta_{10} w^{2}}{105 B i^{2} \eta^{2}}+\frac{8 a^{* 3} F}{105 P e_{z} w^{2} B i^{2} \eta^{2}}\right) \\
& \left.-\frac{2(B i+5)}{5}\left(\frac{8 a^{* 2} F \beta_{12}}{B i \eta}+\frac{a^{* 2} F P e_{z} w \beta_{11}}{3 B i \eta}\right)-6 \beta_{8}^{3}+4 \beta_{9} \beta_{8}\right] \mu_{0, H} .
\end{aligned}
$$

Let us define

$$
\begin{aligned}
\beta_{17}= & \left(1+a^{*} F\right) \beta_{10}-\left(1+a^{*} F\right)^{2}\left(8 \beta_{11}-8 \beta_{12}-\frac{1}{w^{2}}-\frac{8 \beta_{13}}{P e_{z} w}\right) \\
& -\frac{2 a^{* 2} F P e_{z} w^{2}(B i+5) \beta_{10}}{15 B i \eta}+\frac{8}{3}(B i+5) \beta_{15} \\
\beta_{18}= & \left(1+a^{*} F\right)^{3}\left(12 \beta_{13}+P e_{z} \beta_{10}+\frac{12 \beta_{10}}{P e_{z} w^{2}}+\frac{48 \beta_{13}}{P e_{z}^{2} w^{3}}+\frac{6}{P e_{z} w^{4}}-\frac{48 \beta_{11}}{P e_{z} w^{2}} \frac{48 \beta_{12}}{P e_{z} w^{2}}\right), \\
\beta_{19}= & \left(1+a^{*} F\right)\left(\frac{16(B i+5) \beta_{15}}{P e_{z} w^{2}}-\frac{4 a^{* 2} F \beta_{10}}{5 B i \eta}+\frac{24 \beta_{16}}{w}+\frac{2 a^{* 2} F(B i+5) P e_{z} \beta_{13}}{5 B i \eta}\right. \\
& \left.+\frac{16 a^{* 2} F(B i+5) P e_{z} \beta_{11}}{5 B i \eta}\right), \\
\beta_{20}= & \left(\frac{35}{2}+7 B i+B i^{2}\right)\left(\frac{16 a^{*} F \beta_{15}}{21 B i \eta}+\frac{4 a^{* 3} F \beta_{10} w^{2}}{105 B i^{2} \eta^{2}}\right)+\frac{16 a^{* 2} F(B i+5) \beta_{12}}{5 B i \eta} \\
\beta_{21}= & \frac{\left(1+a^{*} F\right)(B i+5) a^{* 2} F}{5 P e_{z}^{2} w^{4} B i \eta}-\frac{3\left(1+a^{*} F\right)^{3}}{P e_{z}^{3} w^{6}}+\frac{8 a^{* 3} F\left(\frac{35}{2}+7 B i+B i^{2}\right)}{315 P e_{z} w^{2} B i \eta}, \\
\beta_{22}= & \frac{15\left(1+a^{*} F\right)^{4}}{P e_{z}^{4} w^{8}}-\frac{2 a^{* 2} F\left(1+a^{*} F\right)^{2}(B i+5)}{5 P e_{z}^{3} w^{6} B i \eta}+\frac{a^{* 4} F{ }^{2}(B i+5)^{2}}{675 P e_{z}^{2} w^{4} B i^{2} \eta^{2}} \\
& -\frac{4 a^{* 3} F\left(1+a^{*} F\right)\left(\frac{35}{2}+7 B i+B i^{2}\right)}{315 P e_{z}^{2} w^{4} B i^{2} \eta^{2}}+\frac{16 a^{* 4} F\left(175+105 B i+27 B i^{2}\right)}{4725 P e_{z} w^{2} B i^{3} \eta^{3}}, \\
\beta_{23}= & \frac{\beta_{6}^{2} \beta_{2}-\beta_{1} \beta_{5}^{2}}{P e_{z} w^{2} \beta_{7}} .
\end{aligned}
$$


Fourth Moment: The fourth moment is expressed as

$$
\begin{aligned}
\mu_{4, H}= & {\left[\frac{\tau_{\text {inj }}^{4}}{5}+\left(\beta_{8}+\frac{2}{P e_{z} w^{2}}\right) \tau_{\text {inj }}^{3}+\left(2 \beta_{17}-\frac{8\left(1+a^{*} F\right)^{2} \beta_{13}}{P e_{z} w^{2}}+4 \beta_{8}^{2}-8 \beta_{9}\right) \tau_{\text {inj }}^{2}\right.} \\
& +\left(12 \beta_{17}\left(\frac{1+a^{*} F}{P e_{z} w^{2}}-\beta_{8}\right)+16 \beta_{21}+12 \beta_{8}^{3}-8 \beta_{9} \beta_{8}+2\left(\beta_{18}+\beta_{19}+\beta_{20}\right)\right. \\
& \left.-\frac{24\left(1+a^{*} F\right) \beta_{8}^{2}}{P e_{z} w^{2}}\right) \tau_{\text {inj }}+8 \beta_{9} \beta_{17}+32 \beta_{8} \beta_{21}+6 \beta_{17}^{2}+16 \beta_{9} \beta_{8}^{2}+24 \beta_{8}^{4}-\frac{16 \beta_{22}}{P e_{z} w} \\
& +\frac{8\left(1+a^{*} F\right)}{P e_{z} w^{2}}\left(6 \beta_{8} \beta_{17}-6 \beta_{8}^{3}-\beta_{18}\right)+8 \beta_{8} \beta_{18}-36 \beta_{8}^{2} \beta_{17}+4\left(1+a^{*} F\right)^{2}(B i+5) \\
& \left(\frac{12 a^{* 2} F}{5 P e_{z} w^{2} B i \eta}\left(\beta_{10}-4 \beta_{11}-4 \beta_{12}\right)+\frac{12 \beta_{15}}{w^{2}}+\frac{P e_{z} \beta_{10}}{5 \beta \eta}+\frac{6 a^{* 2} F}{5 w B i \eta}\left(\beta_{21}+4 \beta_{12}\right)+\frac{4 \beta_{15}}{P e_{z}^{2} w^{4}}\right) \\
& +4\left(1+a^{*} F\right)^{4}\left(\frac{36}{P e_{z} w^{3}}\left(\beta_{13}-\beta_{16}\right)+\frac{P e_{z} \beta_{10}}{4 w}+\frac{4 \beta_{10}}{w}+\frac{3}{w^{2}}\left(4 \beta_{12}-\beta_{10}\right)-\frac{30 \beta_{10}}{P e_{z}^{2} w^{4}}\right. \\
& \left.\frac{120}{P e_{z}^{2} w^{4}}\left(\beta_{11}-\beta_{10}\right)+\frac{120 \beta_{13}}{P e_{z}^{3} w^{5}}+\frac{15 \beta_{21}}{w^{4}}\right)+4\left(1+a^{*} F\right)\left(\frac{35}{2}+7 B i+B i^{2}\right) \\
& \left(\frac{4 a^{* 3} F}{105 B i^{2} \eta^{2}}\left(P e_{z} \beta_{21}+2 \beta_{10}-8 \beta_{11}-8 \beta_{12}\right)+\frac{32 a^{*} F \beta_{15}}{21 P e_{z} w^{2} B i^{2} \eta^{2}}\right)+\frac{4 a^{* 4} F^{2}(B i+5)}{75 B i^{2} \eta^{2}} \\
& \left(2(B i+5)\left(4 \beta_{11}-\beta_{10}\right)+8 \beta_{12}+P e_{z} \beta_{21}+\frac{8(B i+5) \beta_{13}}{P e_{z} w}\right) \\
& \left.+\frac{8\left(175+105 B i+27 B i^{2}\right)}{B i^{2} \eta^{2}}\left(\frac{a^{* 4} F^{2} P e_{z} w^{2} \beta_{10}}{1575 B i \eta}+\frac{4 \beta_{15}}{315 a^{*} F}\right)\right] \mu_{0, H} .
\end{aligned}
$$

\section{References}

[1] Guiochon, G., 2002. Preparative liquid chromatography. J. Chromatogr. A, 965, 129161.

[2] Guiochon, G., Felinger, A., Shirazi, D.G., Katti, A.M., 2006. Fundamentals of preparative and nonlinear chromatography, 2nd ed. ELsevier Academic press, New York.

[3] Bellot, J.C., Condoretm J.S., 1991. Liquid Chromatography Modelling : A Review. Proc. Biochem. 26, 363-376.

[4] Carta, G., 1988. Exact analytical solution of a mathematical model for chromatographic operations. Chem. Eng. Sci. 43, 2877-2883. 
[5] Javeed, S., Qamar, S., Ashraf, W., Seidel-Morgenstern, A., Warnecke, G., 2013. Analysis and numerical investigation of two dynamic models for liquid chromatography. Chem. Eng. Sci. 90, 17-31.

[6] Qamar, S., Abbasi, J.N., Javeed, S., Shah, M., Khan, F.U., Seidel-Morgenstern, A., 2013. Analytical solutions and moment analysis of chromatographic models for rectangular pulse injections, J. Chromatogr. A 1315, 92-106.

[7] Qamar, S., Abbasi, J., Mehwish, A., Seidel-Morgenstern, A., 2015. Linear general rate model of chromatography for coreshell particles: Analytical solutions and moment analysis. Chem. Eng. Sci. 137, 352-363.

[8] Leweke, S., von Lieres, E., 2016. Fast arbitrary order moments and arbitrary precision solution of the general rate model of column liquid chromatography with linear isotherm. J. Comput. Chem. Eng. 84, 350-362.

[9] Felinger, A., Guiochon, G., 2004. Comparison of the kinetic models of linear chromatography. Chromatographia Supplement 60, S175-S180.

[10] van Genuchten, M.Th., Alves, W.J., 1982. Analytical solutions of the one-dimensional convective-dispersive solute transport equation. US Department of Agriculture, Technical Bulletin No. 1661, 151-300.

[11] Batu, V., 1989. A generalized two-dimensional analytical solution for hydrodynamic dispersion in bounded media with the first-type boundary condition at the source. Water Resour. Res. 25, 1125-1132.

[12] Batu V., 1993. A generalized two-dimensional analytical solute transport model in bounded media for flux-type finite multiple sources. Water Resour. Res. 29, 288192.

[13] Leij, F.J., Skaggs, T.H., van Genuchten M.Th., 1991. Analytical solution for solute transport in three-dimensional semi-infinite porous media. Water Resour. Res. 27, 271933. 
[14] Park, E., Zhan, H., 2001. Analytical solutions of contaminant transport from finite one-, two, three-dimensional sources in a finite-thickness aquifer. J. Contam. Hydrol. 53, 41-61.

[15] Chen, J.-S., Liu, Y.-H., Liang, C.-P., Liu, C.-W., Lin, C.-W., 2011. Exact analytical solutions for two-dimensional advection-dispersion equation in cylindrical coordinates subject to third-type inlet boundary conditions. Adv. Water Resour. 34, 365-374.

[16] Chen, J.-S., Liu, Y.-H., Liang, C.-P., Liu, C.-W., Lin, C.-W., 2011. Analytical solutions to two-dimensional advection-dispersion equation in cylindrical coordinates in finite domain subject to first- and third-type inlet boundary conditions. J. Hydrol. $405,522-531$.

[17] Zhang, X., Qi, X., Zhou, X., Pang, H., 2006. An in situ method to measure the longitudinal and transverse dispersion coefficients of solute transport in soil. J. Hydrol. $328,614-9$.

[18] Massabó, M., Cianci, R., Paladino, O., 2006. Some analytical solutions for twodimensional convectiondispersion equation in cylindrical geometry. Environ. Modell. Softw. 21, 6818 .

[19] Massabò, M., Catania, F., Paladino, O., 2011. Exact analytical solutions for twodimensional advectiondispersion equation in cylindrical coordinates subject to thirdtype inlet boundary condition. Adv. Water Resour. 34, 365374.

[20] Qamar, S., Bibi, S., Khan, F.U., Shah, M., Javeed, s., Seidel-Morgenstern, A, 2014. Irreversible and Reversible Reactions in a Liquid Chromatographic Column: Analytical Solutions and Moment Analysis. Ind. eng. Chem. Res. 53, 2461.

[21] Bibi, S., Qamar, S., Seidel-Morgenstern, A., 2015. Irreversible and reversible reactive chromatography: Analytical solutions and moment analysis for rectangular pulse injections. J. Chromatogr. A. 1385, 49-62. 
[22] Qamar, S., Khan, F.U., Mehmood, Y., Seidel-Morgenstern, A., 2014. Analytical solution of a two-dimensional model of liquid chromatography including moment analysis, Chem. Eng. Sci. 116, 576-589.

[23] S. Parveen, S. Qamar and A. Seidel-Morgenstern, 2015. Two-dimensional nonequilibrium model of liquid chromatography: Analytical solutions and moment analysis, Chem. Eng. Sci., 122, 64-77.

[24] Parveen, S., Qamar, S., Seidel-Morgenstern, 2016. A. Analysis of two-Dimensional non-Equilibrium model of linear reactive Ccromatography considering irreversible and reversible reactions. Ind. Eng. Chem. Res. 55, 2471-2482.

[25] Kubin, M., 1965. Beitrag zur Theorie der Chromatographie. Collect. Czech. Chem. Commun. 30, 1104-1118.

[26] Kubin, M., 1965. Beitrag zur Theorie der Chromatographie. 11. Einfluss der Diffusion Ausserhalb und der Adsorption Innerhalb des Sorbens-Korns. Collect. Czech. Chem. Commun. 30, 2900-2907.

[27] Kucera, E., 1965. Contribution to the theory of chromatography: Linear nonequilibrium elution chromatography. J. Chromatogr. A 19, 237-248.

[28] Miyabe, K., Guiochon, G., 2000. Influence of the modification conditions of alkyl bonded ligands on the characteristics of reversed-phase liquid chromatography. J. Chromatogr. A 903, 1-12.

[29] Miyabe, K., Guiochon, G., 2003. Measurement of the parameters of the mass transfer kinetics in high performance liquid chromatography. J. Sep. Sci. 26, 155-173.

[30] Miyabe, K., 2007. Surface diffusion in reversed-phase liquid chromatography using silica gel stationary phases of different C1 and C18 ligand densities. J. Chromatogr. A $1167,161-170$. 
[31] Miyabe, K., 2009. Moment analysis of chromatographic behavior in reversed-phase liquid chromatography. J. Sep. Sci. 32, 757-770.

[32] Ruthven, D.M., 1984. Principles of adsorption and adsorption processes, WileyInterscience, New York.

[33] Schneider, P., Smith, J.M., 1968. Adsorption rate constants from chromatography. AIChE J. 14, 762-771.

[34] Suzuki, M., Smith, J.M., 1971. Kinetic studies by chromatography. Chem. Eng. Sci. $26,221-235$.

[35] Suzuki, M., 1973. Notes on determining the moments of the impulse response of the basic transformed equations. J. Chem. Eng. Japan 6, 540-543.

[36] Wolff, H.-J., Radeke, K.-H, Gelbin, D., 1980. Heat and mass transfer in packed bedsIV use of weighted moments to determine axial dispersion coefficient. Chem. Eng. Sci. 34, 101-107.

[37] Wolff, H.-J., Radeke, K.-H, Gelbin, D., 1980. Weighted moments and the porediffusion model. Chem. Eng. Sci. 35, 1481-1485.

[38] Rice, R.G., Do, D.D., 1995. Applied mathematics and modeling for chemical engineers. Wiley-Interscience, New York.

[39] Koren, B., 1993. A robust upwind discretization method for advection, diffusion and source terms. In C. B. Vreugdenhil, B. Koren, editors, Numerical Methods for Advection-Diffusion Problems, Volume 45 of Notes on Numerical Fluid Mechanics, chapter 5, pages 117-138, Vieweg Verlag, Braunschweig..

[40] Javeed, S., Qamar, S., Seidel-Morgenstern, A., Warnecke, G., 2011. Efficient and accurate numerical simulation of nonlinear chromatographic processes. J. Comput. Chem. Eng. 35, 2294-2305. 
[41] Carslaw, H.S., Jaeger, J.C., 1953. Operational methods in applied mathematics, Oxford University Press, Oxford.

[42] Crank, J., 1975. The mathematics of diffusion, 2nd ed. Clarendon Press, Oxford.

[43] Sneddon, I.H., 1972. The use of integral transforms, McGraw-Hill, New York.

[44] Durbin, F., 1974. Numerical Inversion of Laplace Transforms: An efficient improvement to Dubner and Abate's Method. The Computer Journal 17, 371-376.

[45] Honig, G., Hirdes, U., 1984. A method for the numerical inversion transforms. J. Comput. Appl. Math. 10, 113-132.

[46] Qamar, S., Abbasi, J., Javeed, S., Seidel-Morgenstern, A., 2014. Analytical solutions and moment analysis of general rate model of linear liquid chromatography. Chem. Eng. Sci. 107, 192-205.

Table 1: Standard parameters used in the test problems.

\begin{tabular}{|c|c|c|}
\hline Parameter & Symbol & Value \\
\hline External porosity & $\epsilon_{b}$ & 0.4 \\
Internal porosity & $\epsilon_{p}$ & 0.333 \\
Axial Peclet number & $P e_{z}$ & 600 \\
Radial Peclet number & $P e_{\rho}$ & 15 \\
Henry's constant & $a$ & 4 \\
Intraparticle diffusion resistance & $\eta$ & 2 \\
Biot number & $B i$ & 50 \\
Injected concentration & $C_{0}$ & 1 \\
Time of injection & $\tau_{\text {inj }}$ & 1.0 \\
\hline
\end{tabular}




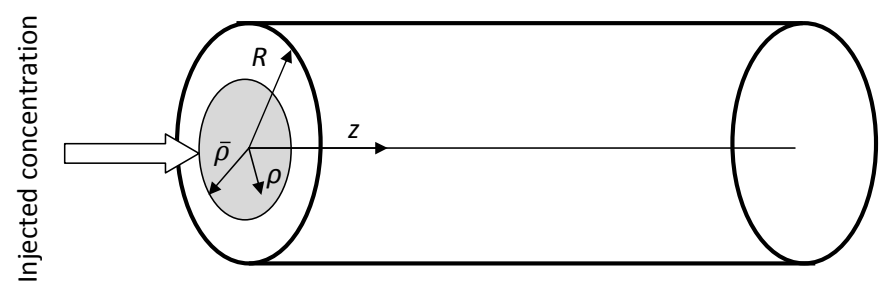

Figure 1: Schematic diagram of a cylindrical chromatographic column packed with unform spherical particles.
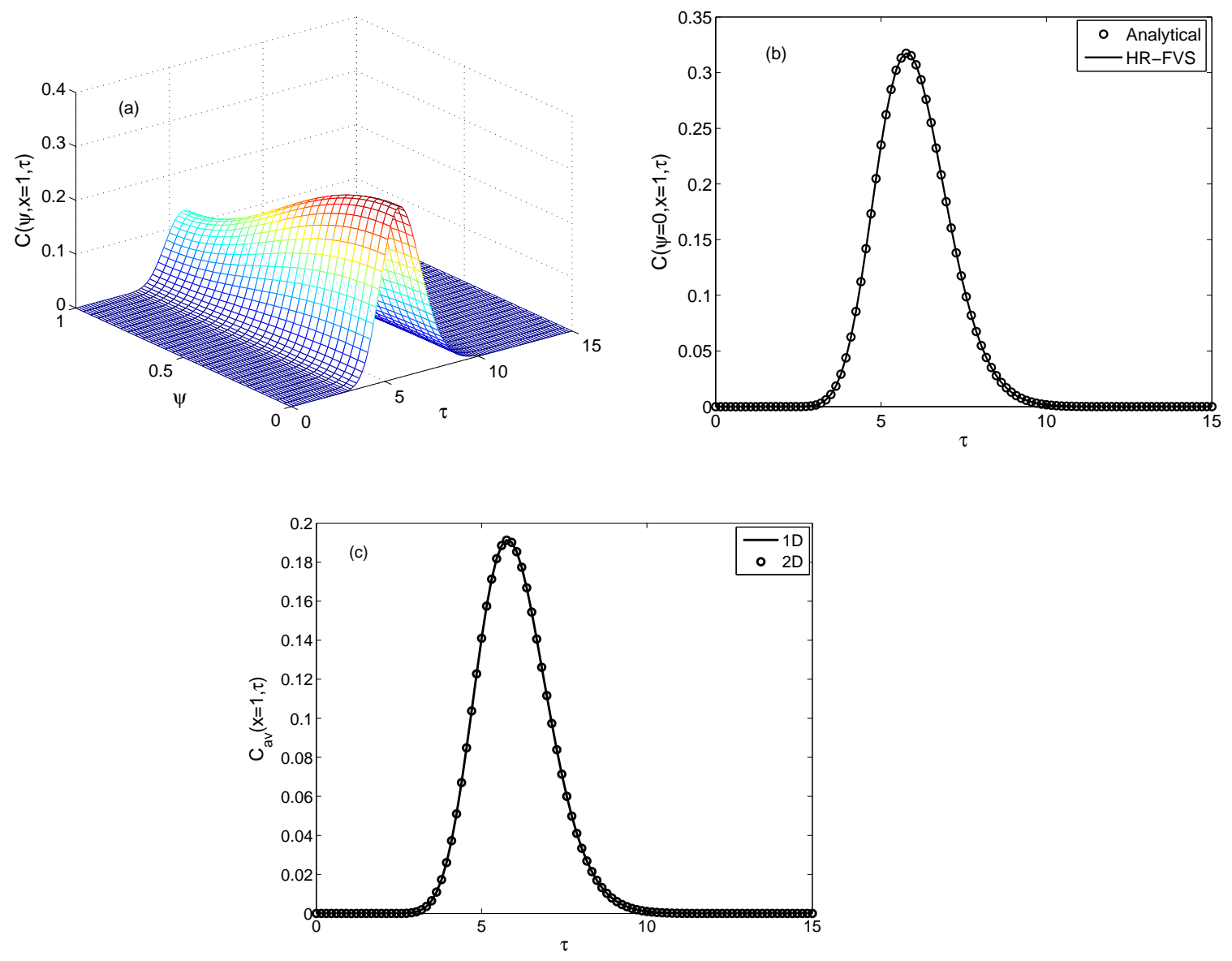

Figure 2: Inner zone injection: Concentration profiles for Danckwerts BCs at $P e_{z}=600, P e_{\rho}=15$, $B_{i}=50$ and $\eta=2$. Plots in (b) show concentration profiles at the column center and plots in (c) give radially averaged concentrations. 

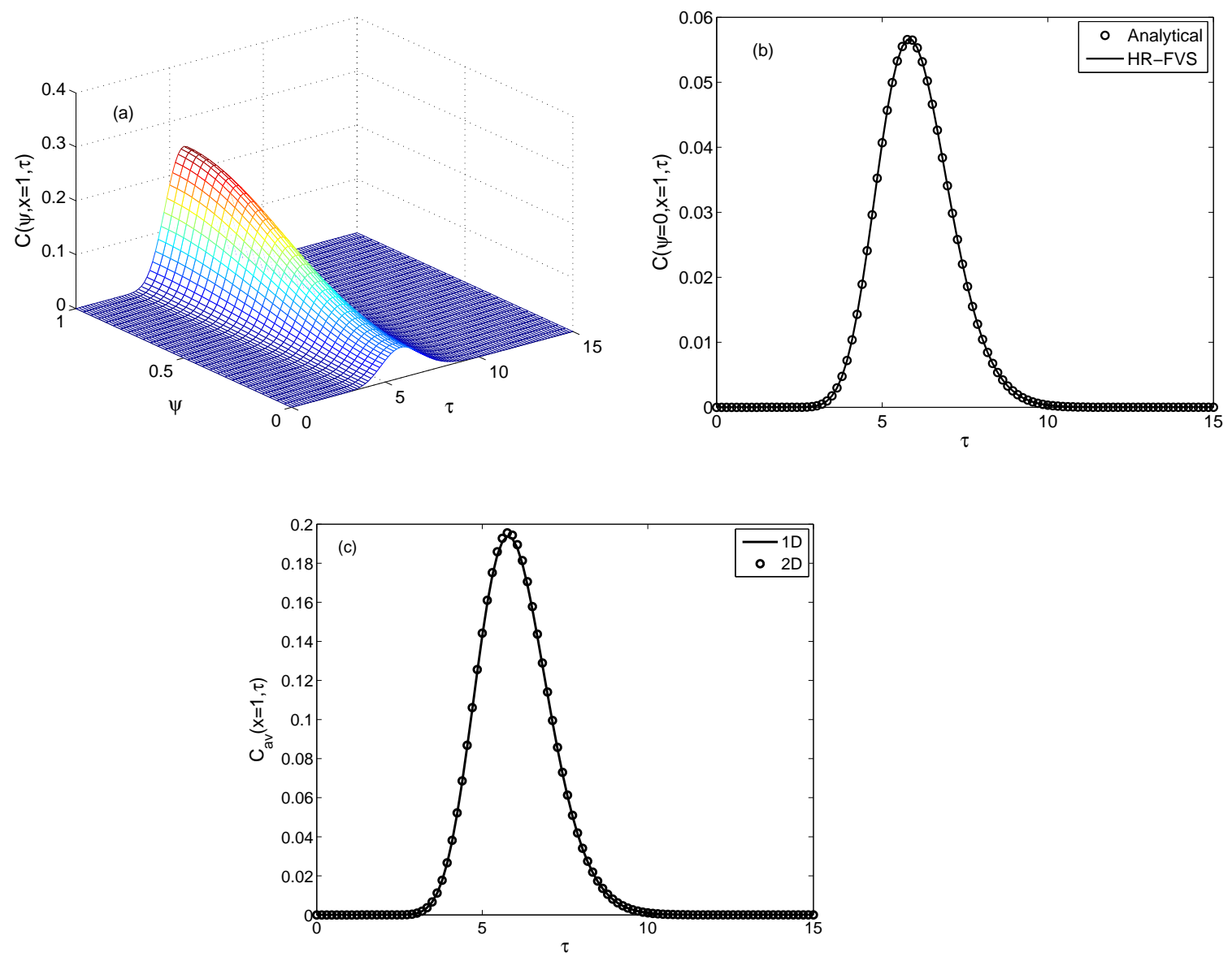

Figure 3: Outer zone injection: Concentration profiles for Danckwerts BCs at at $P e_{z}=600, P e_{\rho}=15$, $B_{i}=50$ and $\eta=2$. Plots in (b) show concentration profiles at the column center and plots in (c) give radially averaged concentrations. 

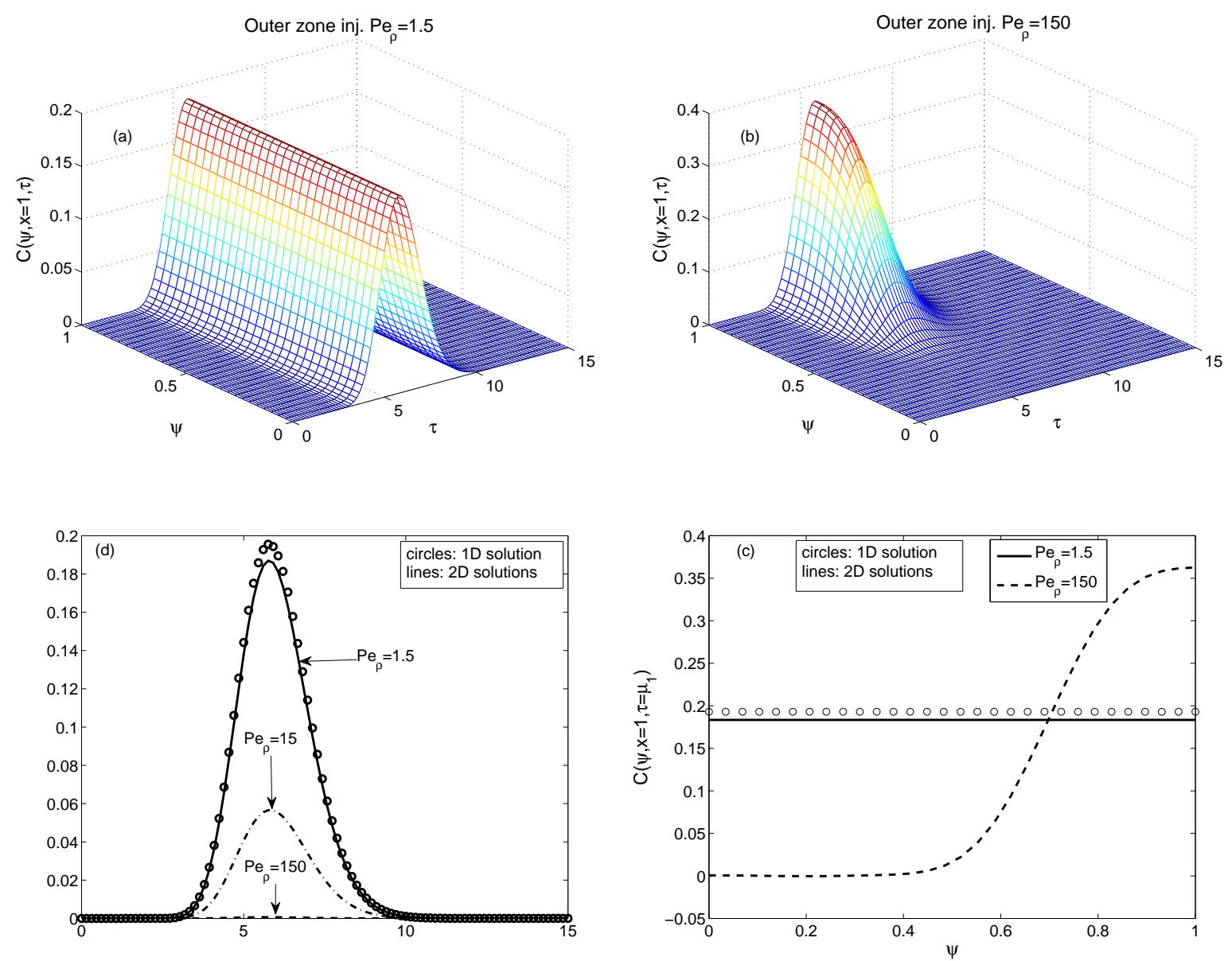

Figure 4: Outer zone injection: oulet concentration profiles for different values of $P e_{\rho}$ using Danckwerts BCs. Here, we have chosen again $P e_{z}=600, B_{i}=50$ and $\eta=2$. Plot (a): 3D plot for $P e_{\rho}=1.5$, plot (b): 3D plot for $P e_{\rho}=150$, plot (c): a comparison of 1D and 2D models solutions in the center of the column, plot (d) a comparison of 1D model solution (circles) with 2D model solutions (lines) for two different $P e_{\rho}$ at the mean retention time. 


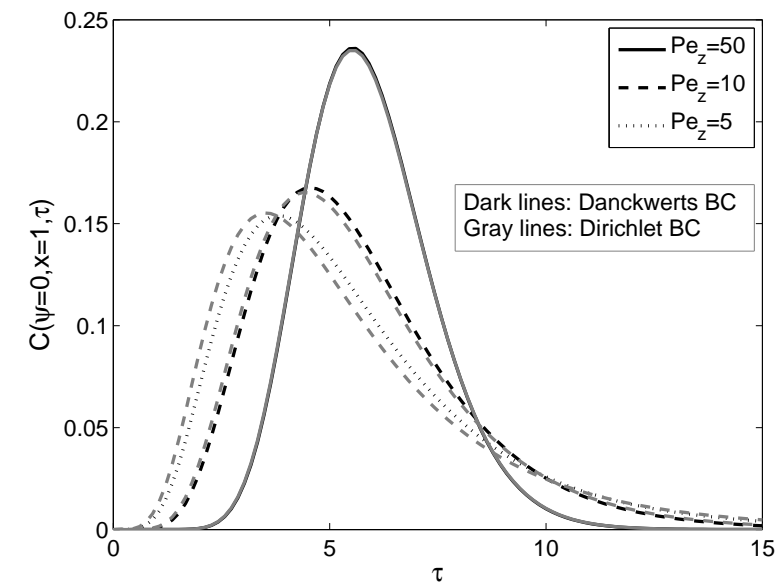

Figure 5: Effects of boundary conditions on the analytical solutions. 

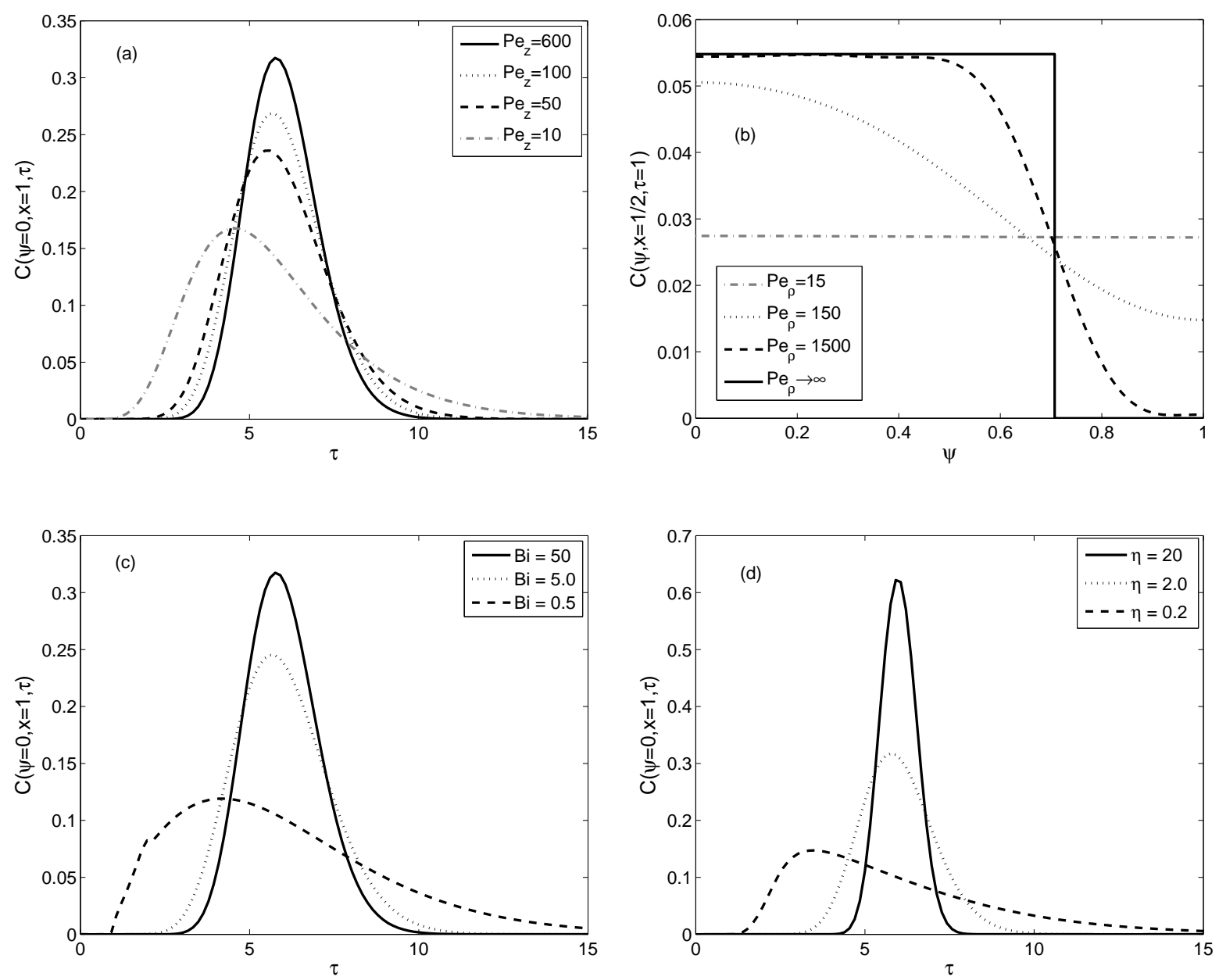

Figure 6: Effects of mass transfer coefficients $B i$ and $\eta$ on the analytical concentration profiles. 

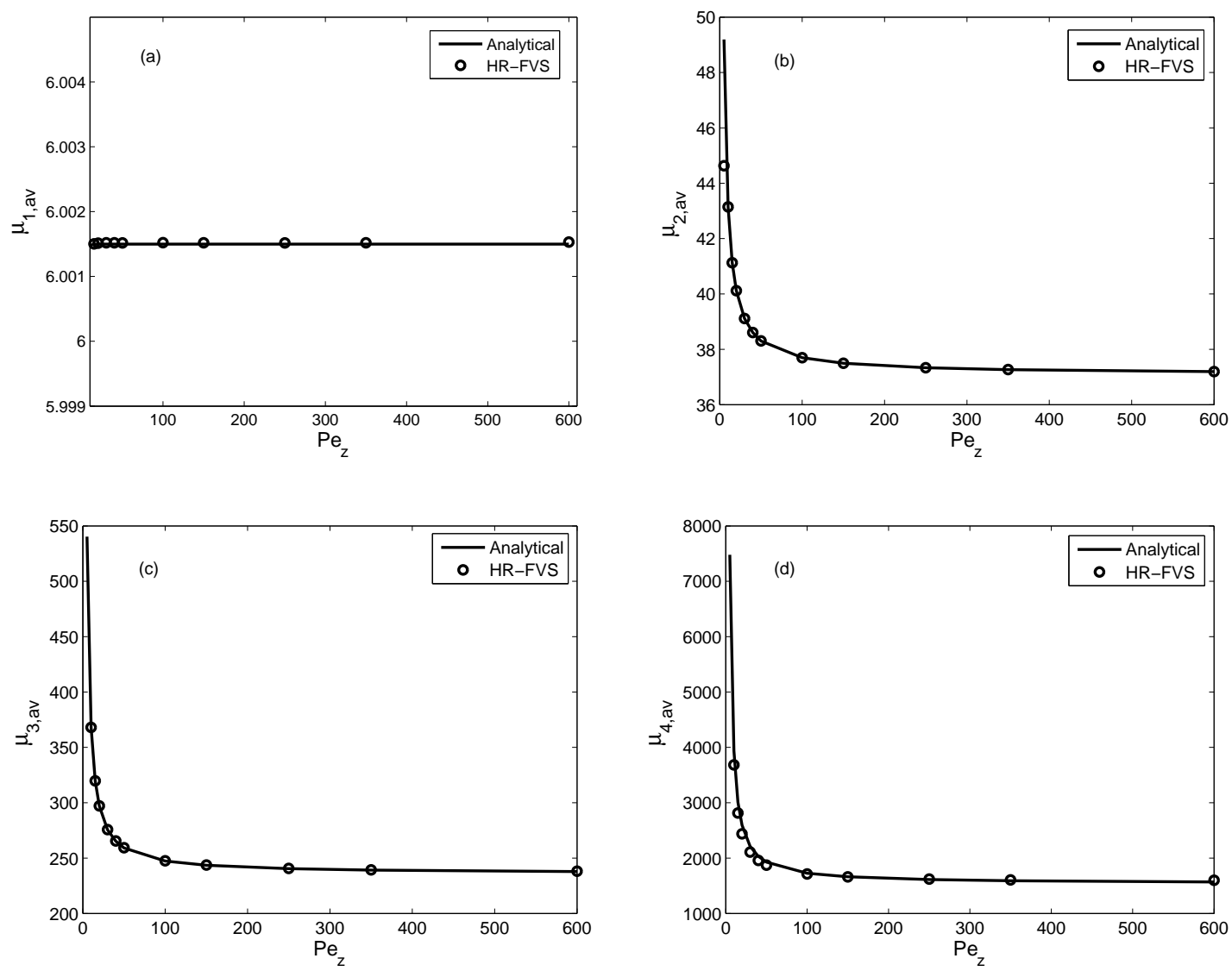

Figure 7: Inner zone injection: Effects of $P e_{z}$ on the averaged moments. 

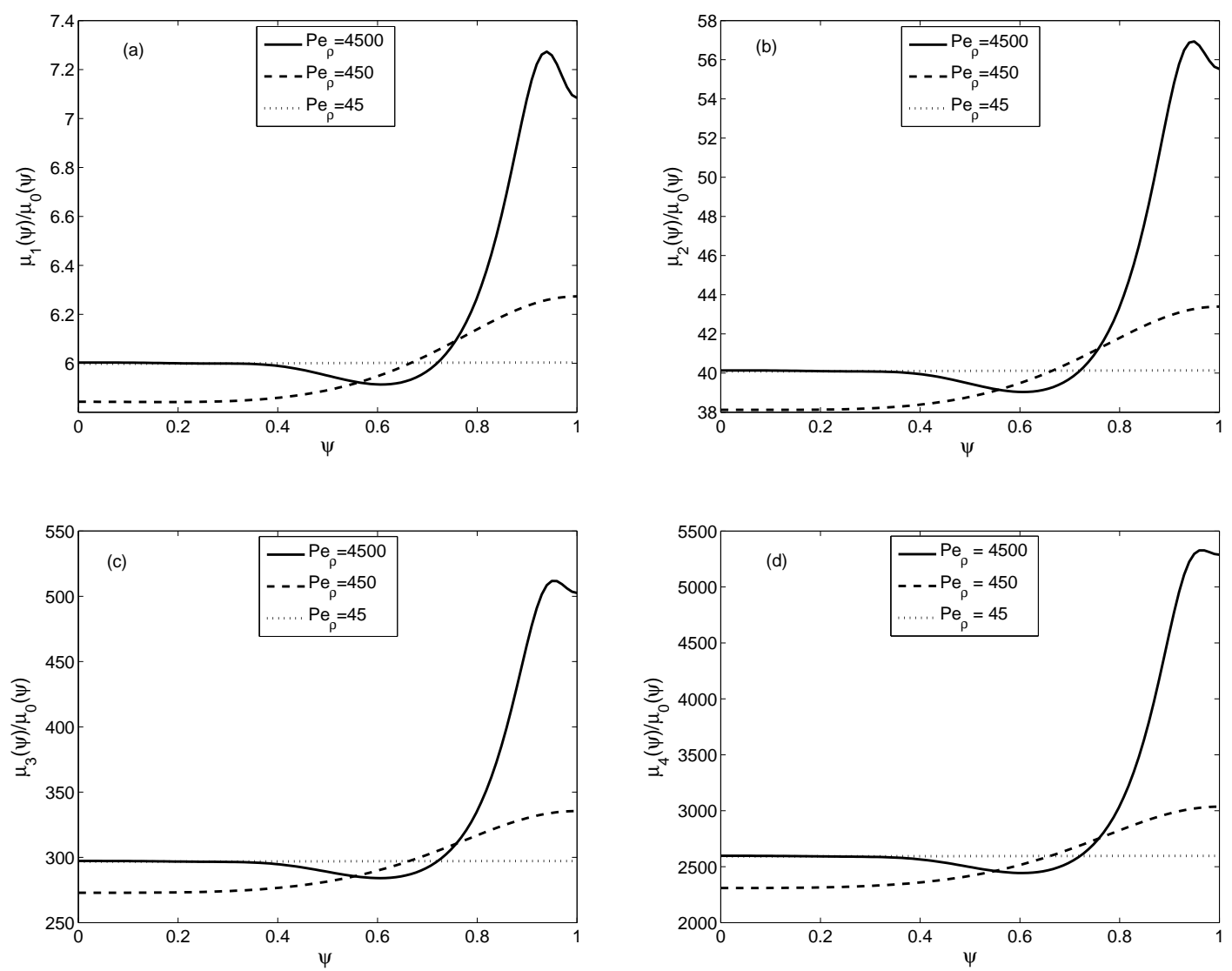

Figure 8: Inner zone injection: Local moments showing the effects of $P e_{\rho}$. 

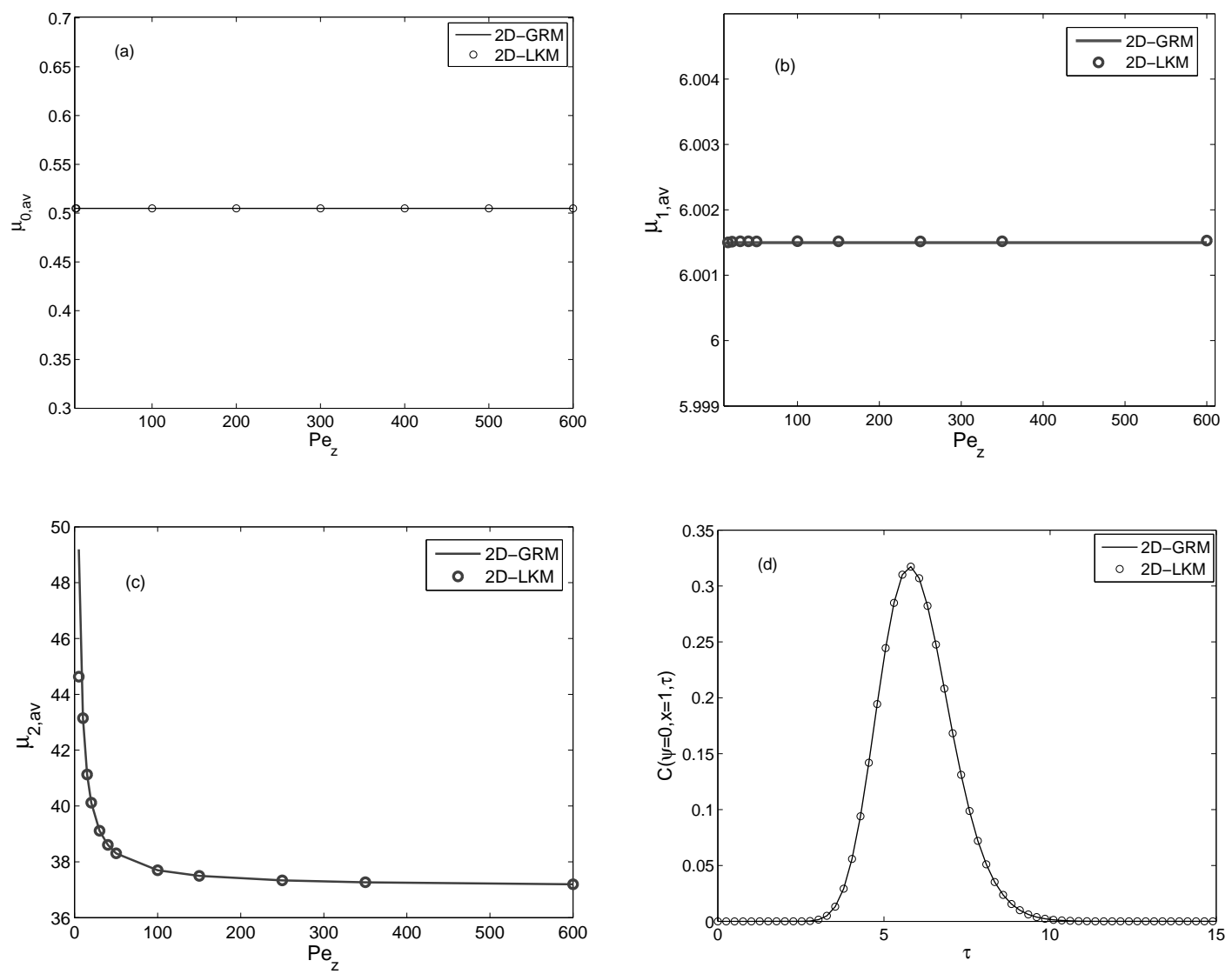

Figure 9: Moments and concentration profiles of 2D-GRM and 2D-LKM when parameters of both models were matched through Eqs. (58) and (59). 